\title{
Coffee consumption and propensity to experience aggressive feelings in provoking and frustrating situations
}

\author{
Anna Zajenkowska ${ }^{1 \cdot A, B, D, E, F, G}$, Konrad S. Fankowski $i^{2 \cdot C, D, E, F}$, Kostas Mylonas $^{3 \cdot A, B}$, Joanna Rajchert ${ }^{1 \cdot D, E, F}$ \\ 1: Department of Social Science, Institute of Applied Psychology, The Maria Grzegorzewska University, Warsaw, Poland \\ 2: Faculty of Psychology, University of Warsaw, Poland \\ 3: Faculty of Psychology, University of Athens, Greece
}

The present study focused on examining whether coffee intake is related to the intensity of aggressive feelings in reaction to various provoking or frustrating situations, along with consideration of sex and culture. Previous studies focused mainly on the dose of caffeine (not the habit of coffee drinking) and aggressive behavior. Many of these studies showed a positive relation between the amount of caffeine consumed and aggression, so it was expected that also habitual coffee consumption would be positively related to propensity for aggressive feelings in situations of provocation and frustration. Participants from Greece $(n=299)$ and Poland $(n=300)$ declared whether they were coffee drinkers (at least 1 mug of coffee per day) and completed the Situational Triggers of Aggressive Responses questionnaire. The results showed that in females, coffee drinkers had higher scores in Sensitivity to Provocation (SP) and Frustration (SF) than nondrinkers, while no differences between drinkers and nondrinkers appeared in males. Moreover, amongst coffee drinkers, females had higher scores in SP and SF than males, while no sex differences were found in nondrinkers. Furthermore, in Poland coffee drinkers had higher SP scores than nondrinkers. Poles also had higher SP scores than Greeks, but only when coffee drinkers were compared.

\section{KEY WORDS}

aggression; coffee; cross-culture; gender; sex

CORRESPONDING AUthor - Anna Zajenkowska, PhD, The Maria Grzegorzewska University, 40 Szczęśliwicka Str., 02-353 Warsaw, Poland, e-mail: azajenkowska@aps.edu.pl

AUthors' CONTRIBUtion - A: Study design - B: Data collection - C: Statistical analysis - D: Data interpretation .

E: Manuscript preparation · F: Literature search · G: Funds collection

to CITE THIS ARTICLE - Zajenkowska, A., Jankowski, K.S., Mylonas, K., \& Rajchert, J. (2015). Coffee consumption and propensity to experience aggressive feelings in provoking and frustrating situations. Current Issues in Personality Psychology, 3(2), 105-111.

RECEIVED 01.04.2015 - REVIEWED 17.05.2015 - ACCEPTED 25.06.2015 • PUBLISHED 29.06.2015 


\section{BACKGROUND}

Aggression is a one of the negative phenomena strongly influencing personal relationships and society as a whole. Thus factors determining its occurrence are of great concern to researchers, practitioners and politicians. Theoretical aggression models, including the General Aggression Model, stress the importance of both individual and situational factors in aggression (Anderson \& Bushman, 2002). Triggering situations, differing among individuals, arouse aggressive feelings that may be suppressed or expressed in a form of aggressive behavior (e.g., verbal or physical). Both suppressing and expressing feelings of aggression have negative outcomes either for the individual experiencing it or for others (Laszlo, Janszky, \& Ahnve, 2010).

The present paper concerns individual differences in propensity to experience feelings of aggression in reaction to various situations. Namely, various situational conditions could be grouped into two types based on the mechanism involved in appraisal of feelings of aggression: frustrating or provoking situations (Lawrence, 2006). Sensitivity to Frustration (SF) is a proneness to feel particularly aggressive in response to having one's goals blocked and to uncontrollable negative events, whereas Sensitivity to Provocation (SP) relates to feeling aggressive in reaction to goading and provocation from others. In general, the experience of feeling aggressive does increase the likelihood of aggressive behavior, for example, individuals high in SP do have an increased tendency to act aggressively towards these who provoke them, but not to those who do not provoke them (Lawrence \& Hutchinson, 2013). Indeed, individuals sensitive to frustration scored higher on anger and hostility and those more sensitive to provocation scored higher on overt physical aggression (Lawrence, 2006).

SP and SF have been related to sex differences (Zajenkowska, Mylonas, Lawrence, Konopka, \& Rajchert, 2014) or personality traits, e.g., individuals higher on SP and SF were also higher on neuroticism and lower on agreeableness (Zajenkowska, Jankowski, Lawrence, \& Zajenkowski, 2013). However, SP and SF seem not to be redundant with personality; for instance, higher SF explained emotional tension after a stressful exam over and above neuroticism (Zajenkowska, Zajenkowski, \& Jankowski, 2015).

Still, little is known about external predictors of the proneness to develop aggressive feelings. External factors are of great interest of researchers, as their presence or absence can be controlled by volitional activity of individuals. Amongst external factors, use of psychoactive substances has received much attention. Explicitly, it has been suggested that such substances as alcohol or caffeine can increase aggressive behavior in an indirect way (Bushman, 1993). That is, when people are under their influence, aggressive feelings are more likely to transform into aggressive behaviors instead of being suppressed. However, the results of the studies are inconsistent, some showing that caffeine diminishes aggressive behavior (Cherek, Steinberg, \& Brauchi, 1983), while others indicate that caffeine leads to higher aggression (Martin et al., 2008). Interestingly, an experimental study by Ferguson, Rule and Lindsay (1982) showed that application of $200 \mathrm{mg}$ of caffeine led to aggression only among those individuals who were also convinced that the drug was administered, but not among those who thought that they took a placebo. In this case caffeine consumption served as an excuse to release hostility. Yet, studies conducted with animals and humans show that the relation between caffeine and aggression, frustration, and irritability is inversely U-shaped and only medium doses increase aggressive behavior (Wilson et al., 2000; Smith \& Tola, 1998). Nevertheless, it is not known in which situations aggressive feelings are more likely to appear in the presence of caffeine. Given that the consumption of coffee is steadily increasing (International Coffee Council, 2012), an answer to the above questions seems increasingly important.

Thus, this study aimed to determine whether coffee consumption is related to propensity to experience aggressive feelings in provoking and frustrating situations. We were interested not in the relation between the amount of coffee drunk per day and SP and SF (though that would also be an interesting question to answer in an experimental study), but whether there are differences between coffee users and non-users in these features. Studies indicate that caffeine consumption can lead to chronically increased anxiety, while acute caffeine effects include facilitation of evoking anxiety by situations likely to trigger it (Hughes, 1996). The individual response to caffeine is, at least partly, biologically determined, particularly by polymorphisms in the adenosine receptor gene (Fredholm, Bättig, Holmén, Nehlig, \& Zvartau, 1999; Rétey et al., 2007). Interestingly, the same gene has also been linked to the level of anxiety (Hamilton et al., 2004), which suggests that anxiety and effects of caffeine share partly the same biological underpinning. In the present study we hypothesize that the above-mentioned interrelationships between caffeine and anxiety may be generalized to propensity to feel aggressive, as the idea that anxiety and anger might be related states, evoked by the same dangerous event, has been discussed for example by Berkowitz and Harmon-Jones (2004). Furthermore, we expected that sex may moderate the association between coffee consumption and feelings of aggression given that females present higher levels of anxiety (Bekker \& van Mens-Verhulst, 2007), SF and SP (Zajenkowska et al., 2014). Finally, since sensitivity to caffeine and anxiety is biologically determined, the differences between countries should not be pronounced regard- 
ing the relation between coffee consumption and SF or SP; therefore country differences were also considered (Poland vs. Greece) to examine the stability of findings cross-culturally.

To sum up, the expectations were that: (1) coffee drinkers would be higher on SP and SF than nondrinkers but (2) female coffee drinkers would be characterized by higher indices of SF and SP than male coffee users.

\section{PARTICIPANTS AND PROCEDURE}

\section{PARTICIPANTS}

Subjects were 599 undergraduates studying either in Poland $(n=300)$ or Greece $(n=299)$. Females constituted $60 \%$ of the total sample and sex distribution in the two countries was similar $\left(\chi_{(1)}^{2}=2.27, p>.05\right)$. Participants were aged between 18 and $56(M=21.30$, $S D=3.50)$ and a two-way ANOVA revealed significant interaction between sex and country regarding age $\left(F(1,590)=10.93, p<.001, \eta_{p}^{2}=.018\right)$. It was found (with Bonferroni adjustment) that males from Poland $(M=22.36, S D=2.15)$ were older than those from Greece $(M=20.08, S D=2.10)(p<.001)$ and for the Greek sub-sample males were younger than females $(M=21.21, S D=5.54)$. For the total sample, $54.2 \%$ of subjects were coffee drinkers and the two countries did not differ in the percentage of coffee users $\left(\chi_{(1)}^{2}=1.10, p=.29\right)$, but females were coffee users more frequently than males $(58.5 \%$ vs. $41.9 \%$, respectively; $\chi_{(1)}^{2}=15.59, p<.001$, with a $p h i$ correlation of .16). Also, a two-way ANOVA revealed that coffee users from Poland $(M=1.74, S D=0.96)$ drank more coffee than those from Greece $(M=1.47, S D=0.86)$ $\left(F(1,300)=6.49, p<.05, \eta_{p}^{2}=.021\right)$.

\section{INSTRUMENTS AND PROCEDURE}

The Situational Triggers of Aggressive Responses scale (STAR; Lawrence, 2006) was used to measure aggression-related feelings. Students completed STAR in groups. The questionnaire consists of 22 items (10 reflect SF, and 12 SP). Participants are presented with 22 situations, and are asked to rate on a 5-point Likert-type scale how each of them typically makes them feel aggressive. The instrument has high internal consistency $(\alpha=.82$ for SP and $\alpha=.80$ for SF) and its validity has been examined previously (Lawrence, 2006; Zajenkowska et al., 2013). The questionnaire was translated for the current study into Polish and Greek by two experts into English and then back-translated by a bilingual person, and approved by the author of the original scale. The internal consistency of the STAR dimensions in the present study was high in both Poland $(\alpha=.78$ for SP and $\alpha=.77$ for SF) and Greece ( $\alpha=.82$ and $\alpha=.80$, respectively). A previous study supported factor equivalence cross-culturally (across Poland, Greece and the UK, Zajenkowska et al., 2014); thus the constructs involved are considered similar across Poland and Greece for the purposes of our study.

Additionally, a question regarding the number of coffee mugs per day was asked and the answers were used to determine coffee drinking status: drinkers (drinking at least one cup a day) vs. non drinkers. The study was approved by the Ethical Review Board of Maria Grzegorzewska Academy of Special Education in Warsaw.

\section{RESULTS}

Relationships between STAR subscales and coffee consumption were analyzed using three-way analysis of covariance (ANCOVA) with country, sex, and coffee consumption status (drinker vs. nondrinkers) as between subject factors, age as a covariate, and each STAR subscale as the dependent variable.

Considering SF, three-way ANCOVA revealed a significant interaction between coffee status and $\operatorname{sex}\left(F(1,576)=9.29, p<.01, \eta_{p}^{2}=.016\right)$, whereas other interactions were statistically non-significant: country by coffee status $\left(F(1,576)=.41, p=.52, \eta_{p}^{2}=.001\right)$, country by $\operatorname{sex}\left(F(1,576)=.31, p=.58, \eta_{p}^{2}=.001\right)$ and country by sex by coffee status $(F(1,576)=.04$, $\left.p=.83, \eta_{p}^{2}=.000\right)$. Analysis of the interaction between coffee status and sex (Figure 1) using Bonferroni adjustment revealed that female coffee drinkers had higher scores in SF than female non-coffee drinkers $(p<.001)$, whereas for males, no difference between

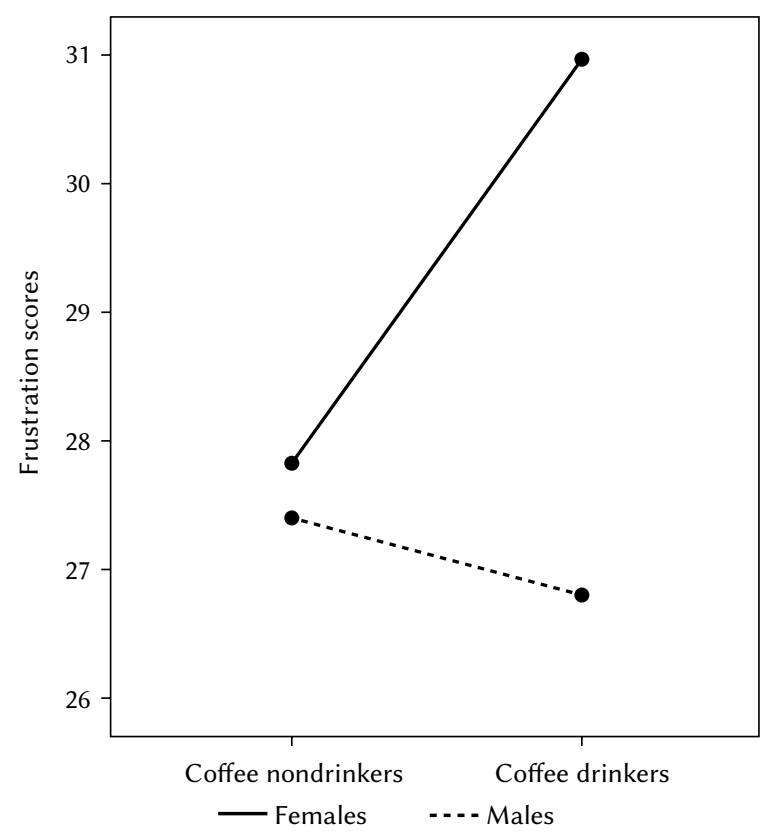

Figure 1. Sensitivity to Frustration as a function of sex and coffee drinking. 
coffee drinkers and nondrinkers regarding SF was observed $(p>.05)$. Moreover, among coffee drinkers, females had higher scores in SF than males $(p<.001)$, while in non-coffee drinkers no sex difference was observed $(p>.05)$. Furthermore, there were significant main effects of coffee status $(F(1,576)=4.29$, $\left.p<.05, \eta_{p}^{2}=.007\right)$ and $\operatorname{sex}(F(1,576)=14.02, p<.001$, $\left.\eta_{p}^{2}=.024\right)$. The effect of country was not statistically significant $\left(F(1,576)=3.23, p=.073, \eta_{p}^{2}=.006\right)$.

Regarding SP, through a three-way ANCOVA design there were significant interactions between coffee status and $\operatorname{sex}\left(F(1,576)=5.28, p<.05, \eta_{p}^{2}=.009\right)$, and between coffee status and country $(F(1,576)=4.63$, $\left.p<.05, \eta_{p}^{2}=.008\right)$. Other interactions were statistically non-significant: country by $\operatorname{sex}(F(1,576)=3.04$, $p=.08, \eta_{p}^{2}=.005$ ) and country by sex by coffee status $\left(F(1,576)=.61, p>.05, \eta_{p}^{2}=.001\right)$. Considering the interaction between coffee status and sex (Figure 2) and using Bonferroni adjustment, it was revealed that for female coffee drinkers higher SP scores were reported than for female non-coffee drinkers $(p<.001)$, while there was no such male difference $(p>.050)$. Moreover, amongst coffee drinkers females had higher Provocation scores than males $(p<.001)$, while in nondrinkers there were no sex differences in Provocation levels $(p=.315)$. Analysis of the interaction between coffee status and country (Figure 3) using Bonferroni adjustment tests revealed that in Poland coffee drinkers had higher Provocation scores than nondrinkers $(p<.010)$, while there were no such difference in Greece $(p=.890)$. Moreover, amongst coffee drinkers Poles had higher Provocation scores than Greeks $(p<.001)$, while in nondrinkers Poles and Greeks did not differ in Provocation levels $(p=.130)$. Also, there were significant main effects observed:

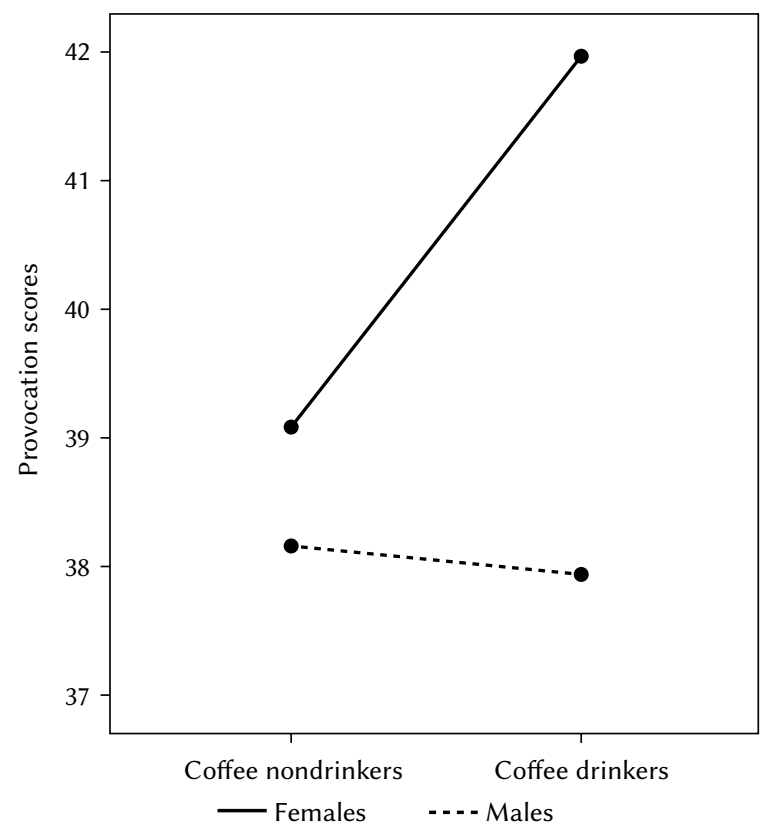

Figure 2. Sensitivity to Provocation as a function of sex and coffee drinking. of coffee status $\left(F(1,576)=3.86, p<.05, \eta_{p}^{2}=.007\right)$, of $\operatorname{sex}\left(F(1,576)=13.48, p<.001, \eta_{p}^{2}=.023\right)$ and of country $\left(F(1,576)=17.30, p<.001, \eta_{p}^{2}=.029\right)$.

\section{DISCUSSION}

It was expected that higher SP and SF would be linked with higher coffee consumption. This hypothesis was confirmed only among females. Female coffee drinkers had higher scores in SP than nondrinkers, and moreover amongst coffee drinkers females had higher scores in SP than males. Much the same relationship was found in regard to SF: female drinkers had higher scores than non-drinkers, and amongst coffee drinkers females had higher scores than males. Furthermore, a difference between the two studied countries was found, but only in SP. Regardless of sex, in Poland coffee drinkers had higher SP scores than nondrinkers, and amongst coffee drinkers Poles had higher SP scores than Greeks.

Considering sex differences, the current study showed that those females who drink coffee are more sensitive to both provocation and frustration. This indicates the possible additive role of coffee consumption and sex for anxiety level. Sawyer, Julia and Turin (1985) pointed out that many studies confirmed that caffeine intake causes changes in arousal, anxiety, and performance and therefore influences human physiology, mood, and behavior. Although small doses increase behavioral activity, cognitive processes, attentiveness and also positive affectivity, high doses may cause or increase anxiety. Smith (2002) stated that consumption of a large amount of caffeine among sensitive individuals (e.g. patients with anxiety dis-

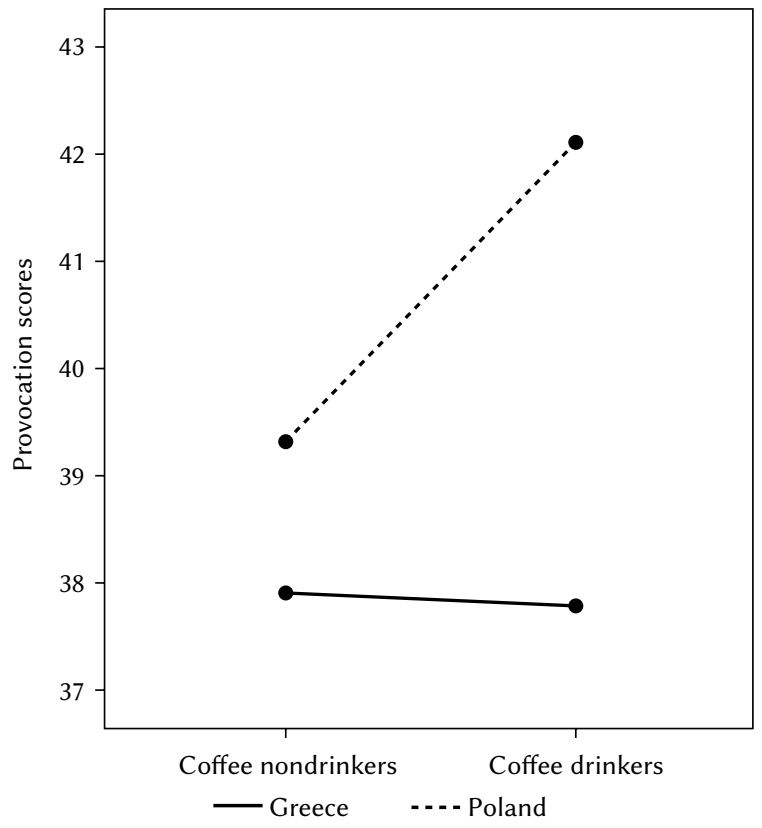

Figure 3. Sensitivity to Provocation as a function of country and coffee drinking. 
orders or in females as in case of the present study) may cause even higher anxiety.

Another explanation for the observed sex differences might be that, though in both men and women caffeine has a similar impact on, for example, blood pressure (BP), it is possible that different hemodynamic mechanisms are responsible for BP responses to caffeine (Hartley, Lovallo, \& Whitsett, 2004). What is more, in some studies women were also more prone to anxiety and had higher SP and SF (Zajenkowska et al., 2014). It was shown that states of anger and anxiety are associated with overlapping regional brain activity (Kimbrel et al., 1999). Anxiety and aggression levels depend on the induction of the same hormone in mice (Patisaul \& Bateman, 2008), and serotonergic disturbances are linked to both aggression and anxiety also in humans (Praag, 1998). Thus, it is probable that the same factors that cause anxiety elevation and caffeine sensitivity (the adenosine sensitivity gene) may produce, maybe indirectly, also high indices of SF and SP among female coffee drinkers.

Another explanation for the sex difference in the relation between coffee use and SP and SF comes from research on magnesium $(\mathrm{Mg})$ metabolism. Women are exposed to a greater $\mathrm{Mg}$ loss, and its absorption and elimination depend on many variables, for instance prolonged stress and excessive menstruation, but also $\mathrm{Mg}$ decrease is related to coffee intake. Furthermore, Mg deficiency may result in increased aggressive reactions (Johnson, 2010). Anger, in turn, leads to liberation of stress hormones and in turn to disorders of the cardiac rhythm. Due to an increased stress level, anger indirectly adds to an unhealthy lifestyle, often including high caffeine consumption. What is more, caffeine and psychological stress have similar physiologic effects (including an increase in blood pressure), and these effects may be additive: blood pressure during stress is significantly higher if caffeine has been consumed (Lane, 1983).

There is one more way the sex differences in coffee drinking and SP and SF might be explained. The results indicated that women were higher on both SP and SF than men, which suggests that they might experience more negative and angry feelings than men do. Also, women were coffee users more frequently than men. At the same time, aggressive behavior is less culturally approved and also less frequent in women, at least for more open and physical forms of aggressive behavior (e.g., Eagly \& Steffen, 1986). Thus, women probably inhibit their aggressive behaviors more, which may produce tension and a more negative mood. We could then hypothesize that women drink coffee for different reasons than men, namely for relaxation or mood improvement. Indeed, some studies show that coffee drinking may boost mood or even lower risk of depression (Lucas et al., 2011). Nevertheless, the benefits of coffee drinking may depend on the level of caffeine intake.
Additionally, our study revealed that in Poland coffee drinkers had higher SP scores than nondrinkers, while no differences between drinkers and nondrinkers were found in Greece. Moreover, Poles had higher SP scores than Greeks, but only when coffee drinkers were compared. Maybe there are some cultural factors that inhibit aggressive feelings in response to provocation among coffee drinkers in Greece (e.g. coffee drinking as social activity in Greece vs. solitary drinking in Poland). These factors require additional investigation.

There are, however, limitations in the current study. Most importantly, we decided to divide all participants into coffee drinkers vs. nondrinkers. In our study, drinking one mug of coffee per day was enough to be in the coffee drinkers group. We did not ask for the coffee strength, type of coffee (e.g., soluble, roasted, espresso) or the capacity of the mugs either, so we were not able to estimate the actual level of caffeine intake. We did not control for other sources of caffeine intake (e.g. energizing beverages or soda) either. One could argue that it would be incorrect to split participants into groups based on such deficient data, since individuals who drink only one cup of coffee each day in the morning probably differ from those who consume numerous cups of coffee during a day. We acknowledge that the amount of caffeine is crucial for eliciting changes in aggression, and larger (but not very big) doses may lead to greater aggression (e.g., Wilson et al., 2000). However, our study was designed to explore the difference between people who do not drink coffee at all (and might not be sensitive to caffeine) vs. those who have such a habit (and probably are sensitive to caffeine). We find it interesting that the habit of regular coffee drinking, irrespective of the amount of caffeine consumption, may be significantly related to SP and SF among women. Further investigations of the relation between aggressive feelings in certain situations and the level of caffeine intake would enrich the results of this study. It would also be useful to control for use of other non-caffeine stimulants, such as smoking or alcohol consumption, that might confound the results. We cannot rule out that the observed effect was due to a specific lifestyle rather than caffeine consumption exclusively. On the other hand, a recent study showed that being a coffee drinker is related to higher depressiveness, whereas drinking other caffeinated drinks or tobacco use had no such effect (Yudko \& McNiece, 2014).

Moreover, we examined university students, which limits our conclusions. Furthermore, the fact that we gathered data based only on a single self-report method makes these data vulnerable to recall bias (as compared to diaries). Finally, as the present study is cross-sectional, a general causal mechanism of the relationship between coffee consumption and aggressive feelings can only be hypothesized. It would be 
interesting to test the relationship of coffee consumption and SF and SP experimentally.

This study does not answer the question about causality. Nevertheless it shows the specific relation between sex, sensitivity to triggers of aggressive feelings (SF and SP) and coffee consumption, which can increase anxiety when taken in excess and indirectly influence health in a negative way. As it was suggested, the relation between coffee consumption and SP and SF may be influenced by the same genes, and although there is a relation between coffee sensitivity and SP and SF, they do not necessarily cause one another. However, this study supports previous findings that show that coffee consumption is related to propensity for aggression, frustration and irritability.

The study was supported by a grant from Maria Grzegorzewska University, Poland.

\section{References}

Anderson, C. A., \& Bushman, B. J. (2002). Human aggression. Annual Review of Psychology, 53, 27-51.

Bekker, M. H., \& van Mens-Verhulst, J. (2007). Anxiety disorders: sex differences in prevalence, degree, and background, but gender-neutral treatment. Gender Medicine, 4, S178-S193.

Berkowitz, L., \& Harmon-Jones, E. (2004). Toward an understanding of the determinants of anger. Emotion, 4, 107-130.

Bushman, B. J. (1993). Human Aggression while under the Influence of Alcohol and Other Drugs: An Integrative Research Review. Current Directions in Psychological Science, 2, 148-152.

Cherek, D. R., Steinberg, J. L., \& Brauchi, J. T. (1983). Effects of caffeine on human aggressive behavior. Psychiatry Research, 8, 137-145.

Eagly, A. H., \& Steffen, V. J. (1986). Gender and aggressive behavior: a meta-analytic review of the social psychological literature. Psychological Bulletin, 100, 309-330.

Ferguson, T. J., Rule, B. G., \& Lindsay, R. C. (1982). The effects of caffeine and provocation on aggression. Journal of Research in Personality, 16, 60-71.

Fredholm, B. B., Bättig, K., Holmén, J., Nehlig, A., \& Zvartau, E. E. (1999). Actions of caffeine in the brain with special reference to factors that contribute to its widespread use. Pharmacological Reviews, 51, 83-133.

Hamilton, S. P., Slager, S. L., De Leon, A. B., Heiman, G. A., Klein, D. F., Hodge, S. E., \& Knowles, J. A. (2004). Evidence for genetic linkage between a polymorphism in the adenosine $2 \mathrm{~A}$ receptor and panic disorder. Neuropsychopharmacology, 29, 558-565.

Hartley, T. R., Lovallo, W. R., \& Whitsett, T. L. (2004). Cardiovascular effects of caffeine in men and wo- men. American Journal of Cardiology, 93, 10221026.

Hughes, R. N. (1996). Drugs which induce anxiety: caffeine. New Zealand Journal of Psychology, 25, 36-42.

Johnson, S. (2010). The multifaceted and widespread pathology of magnesium deficiency. Medical Hypotheses, 56, 163-170.

Kimbrell, T. A., George, M. S., Parekh, P. I., Ketter, T. A., Podell, D. M., Danielson, A. L., \& Post, R. M. (1999). Regional brain activity during transient self-induced anxiety and anger in healthy adults. Biological psychiatry, 46, 454-465.

Lane, J. D. (1983). Caffeine and cardiovascular responses to stress. Psychosomatic Medicine, 45, 447-451.

Laszlo, K., Janszky I., \& Ahnve S. (2010). Anger expression and prognosis after a coronary event in women. International Journal of Cardiology, 140, 60-65.

Lawrence, C. (2006). Measuring Individual Responses to Aggression-Triggering Events: Development of the Situational Triggers of Aggressive Responses (STAR) Scale. Aggressive Behavior, 32, 241-252.

Lawrence, C., \& Hutchinson, L. (2013). The influence of individual differences in sensitivity to provocations on provoked aggression. Aggressive Behavior, 39, 212-221.

Lucas, M., Mirzaei, F., Pan, A., Okereke, O. I., Willett, W. C., O’Reilly, É. J., \& Ascherio, A. (2011). Coffee, caffeine, and risk of depression among women. Archives of Internal Medicine, 171, 1571-1578.

Martin, C. A., Cook, C., Woodring, J. H., Burkhardt, G., Guenthner, G., Omar, H. A., \& Kelly, T. H. (2008). Caffeine use: association with nicotine use, aggression, and other psychopathology in psychiatric and pediatric outpatient adolescents. The Scientific World Journal, 8, 512-516.

Patisaul, H. B., \& Bateman, H. L. (2008). Neonatal exposure to endocrine active compounds or an ER $\beta$ agonist increases adult anxiety and aggression in gonadally intact male rats. Hormones and behavior, 53, 580-588.

Praag, H. V. (1998). Anxiety and increased aggression as pacemakers of depression. Acta Psychiatrica Scandinavica, 98, 81-88.

Rétey, J. V., Adam, M., Khatami, R., Luhmann, U. F. O., Jung, H. H., Berger, W., \& Landolt, H. P. (2007). A genetic variation in the adenosine $\mathrm{A} 2 \mathrm{~A}$ receptor gene (ADORA2A) contributes to individual sensitivity to caffeine effects on sleep. Clinical Pharmacology \& Therapeutics, 81, 692-698.

Sawyer, D. A., Julia, H. L., \& Turin, A. C. (1985). Caffeine and human behavior: Arousal, anxiety, and performance effects. Journal of Behavioral Medicine, 5, 415-439.

Smith, A. (2002). Effects of caffeine on human behavior. Journal of Behavioral Medicine, 19, 111-128. 
Smith, B. D., \& Tola, K. (1998). Caffeine: effects on psychological functioning and performance. In: G. A. Spiller (ed.). Caffeine (pp. 251-299). London: CRC Press.

Trends in coffee consumption in selected importing countries. International Coffee Council. $109^{\text {th }}$ Session, 24-28 September 2012, London, United Kingdom.

Wilson, J. F., Nugent, N. R., Baltes, J. E., Tokunga, S., Canic, T., Young, B. W., \& Hendershot, D. M. (2000). Effects of low doses of caffeine on aggressive behavior of male rats. Psychological Reports, 86, 941-946.

Yudko, E., \& McNiece, S. I. (2014). Relationship between coffee use and depression and anxiety in a population of adult polysubstance abusers. Journal of Addiction Medicine, 8, 438-442.

Zajenkowska, A., Mylonas, K., Lawrence, C., Konopka, K., \& Rajchert, J. (2014). Cross-cultural sex differences in situational triggers of aggressive responses. International Journal of Psychology, 49, 355-363.

Zajenkowska, A., Jankowski, K. S., Lawrence, C., \& Zajenkowski, M. (2013). Personality and individual differences in responses to aggression triggering events among prisoners and non-prisoners. Personality and Individual Differences, 55, 947-951.

Zajenkowska, A., Zajenkowski, M., \& Jankowski, K. S. (2015). The relationship between mood experienced during an exam, proneness to frustration and neuroticism. Learning and Individual Differences, 37, 237-240. 